\author{
Gianluca Cinelli* \\ Goethe-Universität Frankfurt
}

\title{
CRONACA, STORIA E VEROSIMILE. 16 OTTOBRE 1943 E STORIA DELLA COLONNA INFAME
}

\begin{abstract}
L'articolo propone un'analisi comparativa di 16 ottobre 1943 (1944) di Giacomo Debenedetti e Storia della colonna infame (1840) di Alessandro Manzoni. La premessa consiste nel considerare il testo manzoniano come un modello usato da Debenedetti per la sua breve cronaca della razzia del ghetto romano. La comparazione si svolge anzitutto come analisi della retorica narrativa, individuando nelle riflessioni teoriche manzoniane sul rapporto fra vero e verosimile in storia e letteratura la connessione fra la sua opera e quella di Debenedetti. Il secondo punto di confronto è il destino, su cui i due autori divergono: mentre Manzoni lo concepisce come un aspetto della Provvidenza e attraverso la fede trova una consolazione all'ingiustizia, Debenedetti, riconosce nel male soltanto l'assurdità e il cieco caso. La conseguenza teorica e estetica è che entrambi gli autori rifiutano il romanzo come quella forma letteraria ormai incapace di organizzare il destino del personaggio nell'intreccio controllato dal narratore. Pertanto, essi combinano storia, cronaca e verosimile in una narrazione ibrida che non giustifica il male attraverso l'ordine chiuso del racconto ma ne indaga le cause e il senso morale.

Parole chiave: Manzoni, Debenedetti, Non-fiction, storia e letteratura, etica e letteratura, teoria del verosimile.
\end{abstract}

Dopo il silenzio protrattosi fin dal 1938 a seguito della discriminazione razziale, nel settembre del 1944 Debenedetti pubblicò il saggio "Rotolar la botte", ove "racconta uno stato d'animo: quello dell'uomo costretto alla clandestinità e all'inazione, impossibilitato per condizioni esterne o per temperamento a partecipare alla lotta contro il comune nemico; di quest'uomo ci viene detta l'ansia, la trepidazione e, soprattutto, il sentimento di umiliazione e di vergogna per non potere fare di più" (Chiocchetti 2006: 20). Secondo Ciocchetti il breve saggio del 13 settembre 1944 è una sorta di allegoria della resistenza (Ibid) perché Diogene è l'incarnazione dell'intellettuale (Ibid: 21-22). Durante gli anni di silenzio seguiti alla promulgazione delle leggi

*giancin77@yahoo.it 
razziali, Debenedetti si concentrò sempre più sul difficile rapporto dell'individuo con la storia e con il destino. In "Personaggi e destino" scrive che nell'epica moderna le avventure, prive della naturale consequenzialità che pure dovrebbero avere, aggrediscono il personaggio in modo "inquietante": "un divorzio si è consumato tra il protagonista e ciò che gli succede. Si è rotto il rapporto di pertinenza, di legalità tra personaggio e vicenda. Come dire: tra l'uomo e il suo destino" (Debenedetti 1994b: 112). Tale "divorzio" dà adito a una spaccatura nella sensibilità letteraria contemporanea e a due distinte, se non opposte, forme di narrazione:

Nell'epica moderna, non vediamo che due grandi specie, e anch'esse fluttuanti, compenetrate: una che ammette la possibilità di legittimare la vicenda, l'altra che nega questa possibilità. Alla prima diamo il nome di epica della realtà; alla seconda quello di epica dell'esistenza. Nella prima vediamo il personaggio muoversi in mezzo a un mondo con cui c'è ancora la possibilità di un'intesa reciproca. In quest'epica della realtà il personaggio è ancora assistito da qualche cosa, se non altro dalla fiducia in un collegamento tra sé e il mondo. Quello che gli succede, si produrrà dunque come qualche cosa di spiegabile. Nell'epica dell'esistenza, il personaggio è abbandonato da tutto, in mezzo a un mondo anch'esso abbandonato da tutto, e tra i due non è possibile l'intesa, visto che si presentano l'uno all'altro come assurdi. Il mondo ha cessato di rispondere al personaggio; ciò che succede a costui apparirà quindi gratuito (Ibid).

Il termine di riferimento rispetto al quale Debenedetti formula questa diagnosi negativa, e che di lì a pochi anni lo condurrà a esporre la sua celebre Commemorazione provvisoria del personaggio-uomo e a sostituire a questo il personaggio-particella, è il modello del romanzo ottocentesco come definito da Manzoni e consacrato da De Sanctis, i quali avevano rappresentato un'idea di unità (Berardinelli 1996: 862). All'uomo-particella non resta che darsi un nuovo ruolo e con esso il coraggio necessario a sostenerlo: "se la nostra è stata sino ad oggi un'avventura di orfani, facciamoci almeno abbastanza adulti per essere compagni a noi stessi” (Debenedetti 1994c: 127).

16 ottobre 1943 nasce da queste riflessioni. L'opera dedicata alla razzia del ghetto ebraico di Roma non osserva perciò il modello del romanzo e si volge a quello della cronaca, impiegando in modo eclettico diversi registri e strutture del discorso narrativo quali l'analessi, la prolessi, la digressione, il discorso indiretto libero, il dialogo, la citazione, l'ironia e la focalizzazione multipla, al fine di narrare un evento storico prescindendo dal principium individuationis del personaggio-uomo. Il racconto, sotto la direzione di un unico narratore esterno, invece di incentrarsi sul punto di vista di uno o alcuni personaggi-eroi, come nel modello classico del romanzo, assume quello collettivo di una intera comunità unita da null'altro che da un destino comune. L'esito è un racconto senza personaggi popolato di figure su cui il 
destino esercita un'oscura forza motrice, che se da un lato rievoca il mito arcaico, dall'altro si riallaccia a un modello di narrazione storica programmaticamente antiromanzesco qual è Storia della colonna infame.

Alle spalle di 16 ottobre 1943, quindi, si situa come modello Storia della colonna infame piuttosto che la tradizione del romanzo ottocentesco. Per entrambi gli autori gli eventi narrati rappresentano un "soggetto interessante" che spicca dalla continuità dei fatti storici (guerra e peste per Manzoni, guerra e persecuzione razziale per Debenedetti) e che deve essere testimoniato. Entrambi si prefiggono un fine morale: combinando diversi registri retorici e narrativi, essi raccontano le vicende di individui umili e anonimi che di colpo si sono trovati coinvolti in un'esperienza assurda di fronte alla quale i principi etici sono diventati incomprensibili e perfino inutili. Ma i due narratori procedono in modo opposto, perché se Manzoni sceglie di concentrarsi principalmente sulle due figure di Guglielmo Piazza e di Giangiacomo Mora, quasi fino a farne dei personaggi dotati di una psicologia e di un'evoluzione caratteriale (Negri 1972: 27-28), Debenedetti persegue una narrazione in cui le caratterizzazioni dei personaggi sono soppiantate da un discorso collettivo. Similmente, i due narratori ordiscono l'intreccio delle vicende selezionando i singoli episodi e muovendo avanti e indietro il tempo narrativo, aprendo e chiudendo digressioni e rivolgendosi spesso direttamente al lettore. Infine, in entrambe le opere è narrata una "piccola storia", un evento raccontato per penetrare sotto la superficie della storia in cerca di un senso morale al rapporto fra l'uomo e la sua esistenza.

16 ottobre 1943 inizia con la descrizione della vigilia del "Sabbato" ebraico, "che giunge, che giunge come una sposa", con il verbo ripetuto a evocare la gioia di un'intera comunità per l'attesa di quell'evento che ricorda che ogni comunità, ogni singolo ebreo in quel momento condivide la storia e il destino di tutto il suo popolo (Debenedetti 2001: 3). L'atmosfera solenne si spezza di colpo, però, con un'irruzione violenta della "piccola storia": "giungeva invece nell'ex Ghetto di Roma, la sera di quel venerdì 15 ottobre, una donna vestita di nero, scarmigliata, sciatta, fradicia di pioggia" (Ibid: 4). Con l'avversativo e "quella" data precisa si entra nella dimensione spazio-temporale della cronaca, il cui tempo verbale è il presente: "non può esprimersi, l'agitazione le ingorga le parole, le fa una bava sulla bocca" (Ibid). L'urgenza della sua visita al ghetto non sopporta mediazioni da parte del narratore, la donna parla in discorso indiretto libero: "poco fa, da una signora presso la quale va a mezzo servizio, ha veduto la moglie di un carabiniere, e questa le ha detto che il marito, il carabiniere, ha veduto un tedesco, e questo tedesco aveva in mano un lista di 200 capifamiglia ebrei, da portar via con tutte le famiglie" (Ibid). 
Anche l'esordio del capitolo I di Storia della colonna infame annuncia una sciagura che, scaturita dal caso, si addensa in atti, pensieri e sentimenti nei quali si intuisce similmente un destino tragico: "la mattina del $21 \mathrm{di}$ giugno 1630, verso le quattro e mezzo, una donnicciola chiamata Caterina Rosa, trovandosi, per disgrazia, a una finestra d'un cavalcavia che allora c'era sul principio di via della Vetra de' Cittadini [...] vide venire un uomo con una cappa nera, e il cappello sugli occhi, e una carta in mano, sopra la quale, dice costei nella sua deposizione, metteua su le mani, che pareua che scrivesse" (Manzoni 2004: 13-14). L'attacco narrativo è cronachistico e al contempo romanzesco nel modo in cui il narratore dispone con vivida immaginazione la posizione e l'entrata in scena dei protagonisti, come se allestisse una scena. La presenza della "donnicciola", termine spregiativo con cui il narratore anticipa un giudizio morale che sarà coronato dalla citazione virgiliana del capitolo VI (Ibid: 143), ${ }^{1}$ è "disgraziata" perché sarà lei a innescare la catena di ingiustizie e delitti che distruggeranno degli innocenti. L'opinione della donna, espressa attraverso le sue parole in corsivo (citazione dagli atti del processo), costituisce un discorso contrapposto a quello del narratore, che si sottrae alla pretesa del narratore romanzesco onnisciente di dominare gli eventi narrati. Questa collisione fra i due registri, fra le parole di chi crede di sapere ciò che non può dimostrare (Wittgenstein 2011: 8), crea l'atmosfera di tensione e di angoscia che caratterizza entrambe le opere. Manzoni annuncia nell'apparizione dei due personaggi l'imminente propagazione del male come un contagio che travolgerà gli innocenti, primo dei quali proprio Guglielmo Piazza. Il destino che si addensa sulla sua testa è banale e terribile al contempo: vittima innocente della passione collettiva, del "tumulto di chiacchiere" (Manzoni 2004: 19), per salvarsi egli tirerà con sé nell'abisso dell'ingiustizia altri innocenti, e questo farà di lui appunto un "infame". La condanna lo colpisce fin dal primo momento in cui la sua accusatrice lo scorge in strada: lo si vuole untore, infame, e durante il processo egli, per sottrarsi alla tortura, si calunnierà con le sue stesse parole. La colpa dell'innocente, quella stessa che portano nel tempo gli ebrei di Debenedetti, sorge dopo la punizione, anzi in virtù di essa, paradossale, ed è formidabile.

In Debenedetti il destino degli ebrei romani è il medesimo di Israele popolo errante, al quale si oppone l'immagine (ma è solo l'illusione di un momento) della tranquilla quotidianità del ghetto in riva al Tevere descritta nelle prime pagine. Una tranquillità che porta gli sventurati a credere che la Celeste - perché la messaggera ha un'identità, la gente la riconosce e sa che è "una chiacchierona, un'esaltata, una fanatica" (Debenedetti 2001: 5) una pazza a cui non si può dar retta - si sia inventata tutto, o forse abbia

1 "L'infernal dea che alla veletta stava". 
capito male, nonostante le sue proteste veementi che il narratore immagina gridate per la strada: "credetemi! Scappate, vi dico! [...] Vi giuro che è la verità! Sulla testa dei miei figli!" (Ibid: 6). La verità è difficile da scorgere, le passioni che la offuscano sono ugualmente potenti ma opposte a quelle che si agitano in Storia della colonna infame: lì la paura del contagio fa vedere unzioni venefiche ovunque e diventa furore, che reclama un colpevole per placarsi; qui la paura di una storia che si ripete da secoli produce la speranza disperata degli ebrei che si rifiutano di credere a questa Cassandra popolaresca:

Quelle risate, quell'incredulità la esasperano. Comincia a dare in escandescenze e in male parole, come se la minaccia, invece che i tedeschi, fosse stata lei a farla, e ora si offenda di non vederla presa sul serio. Se sapesse cosa inventare, aggraverebbe la dose per vendicarsi, per riuscire finalmente a far paura. Grida, scongiura, si fa venire le lacrime agli occhi, mette le mani sul capo dei bambini, come per proteggerli lei. "Ve ne pentirete! Se fossi una signora mi credereste. Ma perché non ho una lira, perché porto questi stracci..." e nel mostrarli rabbiosamente, li straccia ancora di più (Ibid).

Celeste è per gli ebrei del ghetto un'apparizione d'incubo che deve essere esorcizzata mettendola in ridicolo, facendone uno spauracchio. Eppure il ricordo fresco della minaccia di Kappler, pronunciata a fine settembre, di deportare 200 ostaggi, è lì che preme. La gente vuol trovare nel numero che ritorna una conferma che si tratta di una "storia senza sugo": "la povera gente sa sempre le cose in ritardo e di traverso, ma quel poco che arrivano a sapere credono sempre che sia oro colato. Ormai la minaccia dei 200 ostaggi era scongiurata. I tedeschi saranno dei rascianim, ma sono gente d'onore" (Ibid: 6-7). Qui inizia la digressione, altro lascito manzoniano, dedicata alla mentalità dell'ebreo, con la quale il narratore coglie l'occasione per lasciare il filo della storia e congetturare sulle cause, sulle concatenazioni logiche e illogiche, esplicite e implicite, attraverso cui quella che inizialmente pare solo una favola minacciosa si realizza in modo catastrofico quasi con la tacita collaborazione delle vittime: "contrariamente all'opinione diffusa, gli ebrei non sono diffidenti" (Ibid: 7). In questa breve e nuda sentenza Debenedetti riassume il senso implicito della storia d'Israele: sospettare malafede nel comportamento dei tedeschi "sarebbe stata un'ingiustizia, e per temperamento non vi potevano credere. Mostrar di temere sarebbe stato un polemizzare contro i tedeschi, manifestargli dell'antipatia. E infine sarebbe stato un peccare contro l'Autorità. Perciò, quella sera, gli ebrei risero al messaggio della pazza Celeste" (Ibid: 8). Dopo la digressione inizia la vera "atrocità del dramma", con l'intimazione di Kappler di consegnare cinquanta chili d'oro o di prepararsi alla deportazione di duecento ostaggi. 
Debenedetti descrive il ritorno dei delegati della comunità che hanno consegnato l'oro, il cui sentimento di impotenza e di esaurimento sfugge al cronista e si offre solo obliquamente per mezzo del verosimile: "era in loro quel misto di sollievo e di collasso, che subentra in tutta la persona al termine di una grandissima fatica. Il senso, un po', di chi torna dall'avere accompagnato al cimitero una persona cara, per un cammino lungo e una giornata inclemente, quando si è già estenuati da notti di veglia e di affanno. Ristorarsi, buttarsi in letto, tentare di non pensarci più" (Ibid: 14). Tuttavia questi personaggi sembrano piuttosto delle ombre, come i protagonisti di Storia della colonna infame: Caterina Rosa, il capitano di giustizia, i magistrati, gli imputati stessi agiscono come ombre in un mondo governato da passioni contrastanti. Dei loro sentimenti e delle loro parole Manzoni non ha che i resti consegnatigli dagli atti del processo, e rifiutando di mescolare il verosimile con lo storico, ne fa dei simboli della lotta perpetua dell'umanità sulla terra (Manzoni 1986: 2, 279). In entrambe le opere i protagonisti sono destinati fin da principio alla rovina, in cui il carattere e il destino si illuminano a vicenda: "il destino appare [...] quando si considera una vita come condannata, e in fondo tale che prima è stata condannata e solo in seguito è divenuta colpevole", scrive Benjamin (2004a: 35) e precisa inoltre che "destino è il nesso colpevole di ciò che vive", la "colpa che si eredita nella vita", ossia "una colpa naturale, in cui gli uomini incorrono non con la decisione e l'azione, ma con l'indugio e l'inerzia" (2004b: 177-178).

Gli ebrei di Debenedetti sono destinati alla persecuzione dall'inizio della loro storia; lo sventurato Piazza è destinato a essere un "infame", perché i giudici che lo hanno agguantato non cercano una verità ma pretendono una confessione: "volevano che si confessasse bugiardo una volta, per acquisire il diritto di non credergli, quando avrebbe detto: sono innocente" (Manzoni 2004: 62). In Storia della colonna infame e in 16 ottobre 1943 non troviamo personaggi perché la vicenda non obbedisce alla legge dell'arte (il verosimile) ma alla necessità: $i$ fatti della storia sono incontrovertibili e il male commesso è irreversibile. Scrive Raimondi che "abbandonati da tutti, consapevoli probabilmente di un'ingiustizia che ricevono, senza più essere ascoltati da nessuno, in questo silenzio buio", gli imputati di Manzoni muoiono "nell'ingiustizia totale, senza senso" (2004: 112-113). Quanto più gli imputati tentano di sottrarsi alla macchina che li ha già destinati al supplizio e all'infamia, calunniando se stessi e altri innocenti, tanto più essi realizzano in modo più stringente il loro destino di "infami".

Tuttavia in Manzoni l'ancoraggio della fede dà alla scrittura ancora il nobile compito di redimere. In Debenedetti, invece, l'orizzonte è chiuso, la crudeltà è disperante e si addensa nel colore nero: nera è la veste di Celeste, neri appaiono ai testimoni i camion su cui vengono caricati i deportati (Debenedetti 2001: 31). Il narratore non esprime mai quella fiducia nel vigore 
morale della parola che si coglie invece in Storia della colonna infame, con la sola eccezione della digressione sulla pesatura dell'oro: "nella coscienza dei criminali c'è sempre il senso di una fatalità del castigo" (Ibid: 15). Tuttavia Debenedetti non sembra disposto a credere veramente in una giustizia divina e scrive, perciò, che "più verosimilmente" la ragione del rifiuto di Kappler a emettere una ricevuta fu nel fatto che i tedeschi sapevano che di lì a poco non ci sarebbero stati più ebrei vivi a Roma per impugnarla (Ibid: 31). Su questo punto i due testi divergono radicalmente: Manzoni ammette che dei "fatti atroci dell'uomo contro l'uomo" è responsabile la libera coscienza individuale, e che essa può essere imputata e condannata, scongiurando così la ricaduta in un fatalismo disperato. Debenedetti invece vede solo il destino, imperscrutabile ed enigmatico, che ripete senza posa l'invito a rassegnarsi: nel cuore del racconto della retata il narratore parla degli ebrei che in fila si fanno portare via e dice che "qualche cosa in loro si ricorda di avi mai conosciuti, che erano andati con lo stesso passo, cacciati da aguzzini come questi, verso le deportazioni, la schiavitù, i supplizi, i roghi" (Ibid: 28). Perciò, a differenza del processo agli untori, mosso dalla passione più profondamente umana, la paura, la retata degli ebrei è freddamente lucida, razionale, calcolata, dalla pesa dell'oro alla confisca della biblioteca, fino alla notte angosciosa di sparatoria tutto intorno al ghetto, di cui solo i tedeschi "sapevano la ragione", che forse "era proprio che non ce ne fosse nessuna: l'inferno gratuito, perché riuscisse più misterioso, e perciò più intimidatorio" (Ibid: 22). Il narratore afferma che si trattò di una regia, che i tedeschi amavano insieme con "la teatralità, la solennità nibelungica atra e terrificante" (Ibid: 31), e aggiunge che le SS agirono nel razziare il ghetto romano "con una sorta di rigore professionale, di coscienza del mestiere, piuttosto che stimolati da un preciso accanimento" (Ibid: 38 ), e che quindi è "torto nostro [...] voler cercare una regola nel più spaventoso degli arbitrii” (Ibid: 39$)^{2}$.

È sul piano della retorica narrativa che la somiglianza fra le due opere può essere argomentata, risalendo all'esposizione teorica del rapporto fra "vero positivo" e "verosimile" fatta da Manzoni. Stabilito che nella costruzione del passato assume importanza centrale la nozione di "unità

${ }^{2}$ Debenedetti scrive inoltre: "va notato peraltro che quasi sempre i tedeschi effettuarono le razzie dopo di avere per qualche giorno infuso nelle vittime designate un senso di sicurezza e di tranquillità, mediante false notizie e allentamenti della repressione; era un modo di operare sotto narcosi, per garantirsi una più sollecita e calma riuscita dell'intervento. D'altronde, mentre già la punizione era in atto, essi cercavano di non ribadire il senso della punizione, anzi di mascherarlo. In tutta la giornata i rastrellati ebbero una sola visita dell'ufficiale preposto alla razzia: questi fece la faccia feroce, urlò, sbraitò, ma la visita fu brevissima e non portò visibili peggioramenti della situazione. I giri di vite erano dati subdolamente, le torture erano indirette" (1994a: 80). 
d'azione", con la quale "non si vuol indicare certo la rappresentazione di un fatto semplice e isolato, ma la rappresentazione di un seguito di avvenimenti legati tra loro" (Manzoni 1981a: 61), e quindi un'unità niente affatto arbitraria bensì insita "nella natura stessa della nostra intelligenza" (Ibid: 62), Manzoni sostiene che "per separare alcuni fatti particolari dalla catena generale della storia, e presentarli isolati, bisogna che l'autore sia spinto, sia diretto, da una ragione; bisogna che questa ragione sia insita nei fatti stessi" (Ibid: 63). Tuttavia Manzoni è consapevole del rischio di assolutizzare il concetto di unità come prerogativa della realtà, laddove questo non è che il frutto di speculazione e interpretazione:

Ma esiste realmente tale unità nella natura dei fatti storici? Non vi esiste in maniera assoluta, perché nel mondo morale, come nel mondo fisico, ogni esistenza è a contatto con altre esistenze; ma vi esiste in maniera approssimativa [...]. Che cosa fa dunque il poeta? Trasceglie, nella storia, alcuni avvenimenti interessanti e drammatici, i quali siano così profondamente legati l'uno all'altro, e lo siano così debolmente con ciò che li ha preceduti e seguiti, che la mente, vivamente colpita dal loro reciproco rapporto, si compiaccia a considerarli uno spettacolo unitario, e vivamente si applichi a cogliere tutta l'estensione, tutta la profondità del rapporto che li unisce, a individuare il più nettamente possibile le leggi di causa e di effetto che li governano (Ibid).

Manzoni considera il lavoro dello storico e del poeta uguali dal punto di vista formale: "ricondurre a un punto di vista unitario, e come in virtù di un'unica intuizione, molti fatti separati dalle condizioni del tempo e dello spazio, scartando gli altri fatti che ad essi sono collegati soltanto per coincidenze accidentali" per il primo; trascegliere, "nella storia, alcuni avvenimenti interessanti e drammatici, i quali siano così profondamente legati l'uno all'altro, e lo siano così debolmente con ciò che li ha preceduti e seguiti, che la mente, vivamente colpita dal loro reciproco rapporto, si compiaccia a considerarli uno spettacolo unitario", per il secondo. L'azione unitaria è propriamente "l'insieme e la successione di tutti i fatti rappresentati" (Ibid), ovvero quel che Ricoeur chiama mythos in Tempo e racconto.

Se sul piano formale quindi la narrazione storica e quella poetica si fondano sull'unità d'azione, ciò che le distingue è la materia, alla quale l'elaborazione retorica è strettamente connessa. In Del romanzo storico la riflessione sul rapporto fra vero e verosimile si impernia non più soltanto sul problema estetico dell'unità di azione del racconto, ma sul problema logico dell'“assentimento" della ragione alla verità esposta in un determinato racconto. È con questo argomento che Manzoni separa nettamente storia e poesia, perché alle due forme espressive fa corrispondere due tipi distinti di assentimento, quello storico e quello poetico. Il primo consiste in un "assen- 
timento sui generis, esclusivo, incomunicabile, che si dà alle cose apprese come cose di fatto", il secondo è quello "ugualmente sui generis, esclusivo, incomunicabile, che si dà alle cose apprese come meramente verosimili" (Manzoni 1981b: 206). O si crede nel "vero" o si crede nel "verosimile", e se "conoscere è credere" (Ibid: 199) allora i due piani logici non possono essere mescolati se non con la conseguenza perniciosa di ingannare. Manzoni legittima la pretesa di verità tanto della storia quanto dell'invenzione, perché l'effetto dell'arte è assoluto in quanto riposa sul riconoscimento di una "incorruttibile entità" dell'oggetto artistico, una vera e propria "aura". Pertanto la verità appartiene tanto alla storia, cioè al "vero positivo", quanto alla poesia, cioè al verosimile, e ciò in virtù di un assentimento logico che non ammette antinomie o ambiguità: o la ragione assentisce "storicamente" credendo che gli oggetti che conosce siano "cose di fatto", oppure assentisce poeticamente, credendo che gli oggetti che conosce siano "cose meramente verosimili", possibili. È vera quindi quella narrazione che "proponendosi un fine sensato, adopra i mezzi più adatti a ottenerlo fin dove si può", e quindi si impegna a fornire una cognizione "non perfetta, ma effettiva" dei fatti del passato, anche là dove non può procedere se non per via di congettura (Ibid). Qui soltanto è autorzzata la presenza del verosimile nel discorso storico come possibile mezzo di argomentazione:

Anche del verosimile la storia si può qualche volta servire, e senza inconveniente, perché lo fa nella buona maniera, cioè esponendolo nella sua forma propria, e distinguendolo così dal reale. E lo può fare senza che ne sia offesa l'unità del racconto, per la ragione semplicissima che quel verosimile non entra a farne parte. È proposto, motivato, discusso, non raccontato al pari del positivo, e insieme col positivo, come nel romanzo storico. E non c'è nemmeno pericolo che ne rimanga offesa l'unità del componimento, poiché qual legame più naturale, qual più naturale continuità, per così dire, di quella che si trova tra la cognizione e l'induzione (Ibid)?

Manzoni espone un'estetica della narrazione referenziale fondata sull'assentimento logico alla positività dei fatti esposti, di conseguenza il verosimile in quanto argomentazione di una verità possibile ma non necessaria si presta come strumento nelle mani dello storico là dove questi si trova nell'impossibilità di esporre con forza di evidenza i fatti:

Quando la mente riceve la notizia d'un positivo che ecciti vivamente la sua attenzione, ma una notizia tronca e mancante di parti o essenziali, o importanti, è inclinata naturalmente a rivolgersi a cose ideali che abbiano con quel positivo, e una relazione generale di compossibilità, e una relazione speciale o di causa, o d'effetto, o di mezzo, o di modo, o d'importante concomitanza, che ci hanno dovuta avere le cose reali di cui non è rimasta la traccia. È una parte della miseria dell'uomo il non poter conoscere se non qualcosa di ciò che è stato, anche nel suo piccolo mondo; ed è una 
parte della sua nobiltà e della sua forza il poter congetturare al di là di quello che può sapere. La storia, quando ricorre al verosimile, non fa altro che secondare o eccitare una tale tendenza. Smette allora, per un momento, di raccontare, perché il racconto non è, in quel caso, l'istrumento bono, e adopra in vece quello dell'induzione: e in questa maniera, facendo ciò che è richiesto dalla diversa ragione delle cose, viene anche a fare ciò che conviene al suo novo intento. Infatti, per poter riconoscere quella relazione tra il positivo raccontato e il verosimile proposto, è appunto una condizione necessaria, che questi compariscano distinti (Ibid: 213-214).

Per Manzoni il momento della congettura, che in termini retorici si caratterizza come uno scarto critico-digressivo dal racconto, è una deviazione del ragionamento che in modo circolare tenta di connettere premesse e conclusioni, o cause ed effetti, che non sono immediatamente evidenti. Tuttavia la congettura prevede una pre-comprensione, un'anticipazione interpretativa di ciò che si intuisce, date certe premesse, essere un possibile sviluppo con certe possibili conseguenze. Lo storico deve quindi saper "scegliere", "scartare", "accozzare", confrontare", "dedurre" e "indurre" (Ibid: 217).

Ciò che Manzoni descrive in termini speculativi in Del romanzo storico e mette in pratica con la scrittura di Storia della colonna infame si riscontra anche in 16 ottobre 1943, sebbene con una differenza nelle premesse: se infatti la vicenda del processo è nota a Manzoni grazie ai documenti (atti, lettere e cronache), la razzia del ghetto romano è appresa da Debenedetti in modo del tutto differente, come racconto orale diffuso fra la popolazione, anonimo e collettivo al contempo. Mentre Manzoni ha quindi appigli concreti su cui congetturare, fra cui perfino le parole pronunciate dai protagonisti del processo, Debenedetti non ha che un canovaccio, un'atmosfera e frammenti di una storia che assume contorni talora incerti nelle parole dei pochi testimoni (la signora S., il cui racconto "senza dubbio ripetuto molte volte nel corso di questi mesi, sarà certo un po' ricostituito, con un ordine nell'incastro dei fatti nella sequenza dei tempi, che forse la vita non ebbe" (Debenedetti 2001: 32), e tanti altri, perlopiù anonimi, fino all'ultima persona che vide gli ebrei vivi sul treno fermo nella stazione di Firenze, il macchinista Zazza). Per Debenedetti vale ironicamente quel che Manzoni scrive in Storia della colonna infame: egli fa "come que' ragni, che attaccano i capi del loro filo a qualcosa di solido, e poi lavoran per aria" (Manzoni 2004: 128). Ma l'intenzione per entrambi gli autori resta quella di aderire al vero quanto più possibile, di non inventare e di limitare il lavoro dell'immaginazione al riempimento dei vuoti e delle oscurità (Riccardi 1990: 174). Manzoni si domanda come poterono i giudici non vedere le inverisimiglianze del racconto della testimone-accusatrice Caterina Rosa, la quale "potrebb'esser benissimo che [...] avesse parlato d'una penna da lei vista davvero in mano dello sconosciuto; e ognuno indovina troppo fa- 
cilmente qual altra cosa poté esser da lei battezzata per vasetto; ché, in una mente che non vedeva che unzioni, una penna doveva avere una relazione più immediata e più stretta con un vasetto, che con un calamaio" (Manzoni 2004: 19) E ancora il narratore di Storia della colonna infame si domanda come si possa passare da un sospetto alla certezza di un crimine in assenza di indizi e prove, e per farlo non può che congetturare, immaginare uno stato psicologico collettivo, un'atmosfera di paura che acceca le menti:

Il sospetto e l'esasperazione, quando non siano frenati dalla ragione e dalla carità, hanno la trista virtù di far prendere per colpevoli degli sventurati, sui più vani indizi e sulle più avventate affermazioni. [...] L'essere il primo che trovavan lì nelle vicinanze; l'essere sconosciuto, e non dar di sé un conto soddisfacente: cosa doppiamente difficile quando chi risponde è spaventato, e furiosi quelli che interrogano; l'essere indicato da una donna che poteva essere una Caterina Rosa (Ibid: $21-22)$.

Tutto ciò, immagina il narratore, crea assassini là dove la paura vuol vederli, e i giudici, nient'affatto scellerati o ignoranti, seguono nel solco scavato da quelle passioni:

E non paia strano di vedere un tribunale farsi seguace ed emulo d'una o due donnicciole; giacché, quando s'è per la strada della passione, è naturale che più ciechi guidino. Non paia strano il veder uomini i quali non dovevan essere, anzi non eran certamente di quelli che vogliono il male per il male, vederli, dico, violare così apertamente e crudelmente ogni diritto; giacché il credere ingiustamente, è strada a ingiustamente operare, fin dove l'ingiusta persuasione possa condurre (Ibid: 65).

Il verosimile subentra in ogni momento in cui non è possibile conoscere positivamente le premesse o i passi intermedi di un ragionamento o di una situazione (Ibid: 73), come quello che porta Guglielmo Piazza a calunniare l'innocente Giangiacomo Mora per ottenere l'impunità offertagli dal tribunale: "quello che passò in quell'abboccamento, nessuno lo sa, ognuno se l'immagina a un di presso. [...] Non pare però punto probabile che il Piazza abbia chiesto lui l'impunità [...] ed è ben più credibile, che, per fargli fare quel primo, così strano e orribile passo, per tirarlo a calunniare sé e altri, l'auditore gliel'abbia offerta" (Ibid: 75). La retorica della congettura, la tensione fra non sapere e immaginare, mette in questione un'evidenza che è tale per il buon senso e per la coscienza morale ma non per la logica, che non coglie il nesso referenziale. E così si spalanca un abisso davanti al quale lo storico esita in dubbio se arrestarsi e sospendere il giudizio, o adottare gli strumenti del poeta e affrontare quell'oscurità per cavarne la verità morale che cerca: 
Ma chi può immaginarsi i combattimenti di quell'animo, a cui la memoria così recente de' tormenti avrà fatto sentire a vicenda il terror di soffrirli di nuovo, e l'orrore di farli soffrire! A cui la speranza di fuggire una morte spaventosa, non si presentava che accompagnata con lo spavento di cagionarla a un altro innocente! Giacché non poteva credere che fossero per abbandonare una preda, senza averne prima acquistata un'altra almeno, che volessero finire senza una condanna (Ibid: 76).

A differenza del romanzo, dove la coscienza dei personaggi è un libro aperto per il narratore onnisciente, qui essa rimane impervia e buia, un mistero in cui il narratore non può penetrare con passo sicuro ma solo brancolare. Ma se la coscienza degli imputati trascinati con il dolore e la paura a calunniare se stessi e altri è un enigma, ancor più impenetrabile resta la coscienza dei giudici, nella quale solo Dio può affondare lo sguardo con certezza e capire, discernere: "cosa facevano, trovandosi a tal punto, de' giudici ai quali la passione non avesse pervertita, offuscata, istupidita la coscienza? Si spaventavano d'essere andati (foss'anche senza colpa) tanto avanti; si consolavano di non essere almeno andati fino all'ultimo, all'irreparabile affatto; si fermavano sull'inciampo fortunato che gli aveva trattenuti dal precipizio" (Ibid: 105). Invece la storia tramanda tutt'altro, una vicenda in cui la caparbia ostinazione di giudici che pur potendo vedere (o forse vedendo) l'ingiustizia che commettevano non si ritrassero e assassinarono molti innocenti come colpevoli di ciò che non avevano commesso, sebbene mandassero infine assolto Padilla, imputato come mandante del delitto e capo della cospirazione, un'assoluzione che di fatto scagionava tutti gli altri imputati già giustiziati da tempo: "di tanti orrori fu cagione la debolezza... che dico? L'accanimento, la perfidia di coloro che, riguardando come una calamità, come una sconfitta, il non trovar colpevoli, tentarono quella debolezza con una promessa illegale e frodolenta" (Ibid: 131), cioè indussero Piazza a calunniare i suoi "complici" promettendogli una salvezza illegale che, di fatto, gli revocarono. L'argomentazione verosimile si pone così come punto di giuntura fra quella storica e quella morale, poiché molto spesso la congettura che il narratore avanza sottende anche una questione o un giudizio morali.

Debenedetti costruisce il racconto sulla base di testimonianze orali e la contemporaneità del soggetto gli permette di affrontarlo senza ulteriori premesse e spiegazioni. Il suo pubblico sa cosa sia stata l'occupazione tedesca a Roma e altrove, le persecuzioni razziali sono una realtà del momento (nel tempo in cui Debenedetti scrive e pubblica il libello la guerra prosegue in Europa e in Italia settentrionale) che non richiede la ricostruzione di un contesto storico di supporto per mezzo di digressioni come quelle che invece Manzoni intercala in Storia della colonna infame sulla criminologia del Seicento, sulla pratica della tortura e sulla Milano del 1630 colpita 
dalla peste. Ciò fa sì che il racconto di Debenedetti appaia, rispetto a quello manzoniano, meno frastagliato e movimentato, perché rimane sempre aderente alla cronaca, prediligendo il passato remoto come tempo dominante della narrazione, ma adottando il presente nelle pagine drammatiche della descrizione della retata, con un netto accorciamento della distanza storica ed emotiva rispetto agli eventi e l'assunzione di un ritmo quasi visivo o cinematografico. Rispetto a Storia della colonna infame il verosimile ricopre in 16 ottobre 1943 uno spazio più esteso, perché il narratore ha un supporto meno sicuro su cui imbastire il discorso e deve di conseguenza "riempire" con un intervento più sostanzioso una vicenda che rischierebbe di essere ridotta a due o tre episodi salienti.

Dopo la digressione sul destino degli ebrei, Debenedetti salta all'indietro narrando il proemio dell'intimazione di Kappler a consegnare cinquanta chili d'oro per salvare il ghetto dalla deportazione (Debenedetti 2001: 9). Il fatto viene "rimpolpato" dal narratore che lo estende per alcune pagine. Nel dipanare le premesse della retata Debenedetti ricorre spesso al verosimile, soprattutto quando narra la spoliazione del tempio e della biblioteca rabbinica da parte delle SS e del misterioso personaggio che esegue l'operazione:

Accompagnato anche lui da una scorta di SS, al vederlo si direbbe un ufficiale tedesco come tutti gli altri, con quel più di arroganza che gli dà l'appartenere a una "specialità" privilegiata e tristemente famosa. Tutto divisa, anche lui, dalla testa ai piedi: quella divisa attillata, di un'eleganza schizzinosa, astratta e implacabile, che inguaina la persona, il fisico ma anche e soprattutto il morale, con un ermetismo da chiusura-lampo. È la parola verboten tradotta in uniforme: proibito l'accesso all'uomo e all'individuale passato che vive in lui, che è la sua storia e la sua più vera "specialità" di creatura di questo mondo; proibito vedere altro che questo suo "presente" rigoroso, automatico, intransigentemente reciso (Ibid: 16).

L'accesso al personaggio è precluso e restano solo i suoi gesti che il narratore immagina e fissa sulla pagina: "con mani caute e meticolose, da ricamatore di fino, palpa, sfiora, carezza papiri e incunaboli, sfoglia manoscritti e rare edizioni, scartabella codici membranacei e palinsesti. La varia attenzione del tocco, la diversa cautela del gesto sono subito proporzionate al pregio dei volumi" (Ibid). Al narratore è preclusa la via all'interiorità di questo personaggio, proprio come la era al narratore di Storia della colonna infame: se Manzoni aveva trovato le parole e talvolta i gesti di quegli uomini scritti negli atti del processo, e a partire da quello aveva immaginato delle coscienze opache e complesse, Debenedetti non ha niente se non l'immaginazione per far intuire che l'ufficiale è uno studioso, un amante infelice e crudele della cultura ebraica, un "egregio cultore di paleografia e filologia 
semitica", come "più tardi si seppe" (Ibid: 17). Per un istante quest'uomo si illumina, irradia una personalità, ma poi "un colpo secco della chiusuralampo, e la divisa ha rinserrato il semitologo, che è ridivenuto un ufficiale delle SS" (Ibid). Infine il racconto torna lì dov'era iniziato, alla notte del 15 ottobre, al passato subentra il presente, e come la prospettiva si stringe attorno al ghetto assediato dagli spari incomprensibili dei soldati tedeschi che lo stringono con la paura, i confini di questo spazio diventano quelli di un'umanità che si scopre in trappola:

I coraggiosi si avvicinano alle finestre. Pallottole e schegge sibilano e guaiscono a pochi centimetri dalle persiane, si piantano nei vecchi intonaci delle facciate. [...] Dagli elmetti, si direbbe che sono tedeschi; ma l'occhiata è stata rapida, non è prudente rimanere presso la finestra. Ora i jorbetín si sono messi anche a urlare e schiamazzare: voci e grida squarciate, colleriche, sarcastiche, incomprensibili. Che vogliono? Con chi ce l'hanno? Dove vanno? Nelle case ormai sono tutti in piedi. I vicini si riuniscono per farsi coraggio, e viceversa non riescono che a farsi paura a vicenda (Ibid: 20).

In queste pagine Debenedetti si allontana dalla cronaca, lascia che l'immaginazione disegni in aria i gesti e faccia risuonare pensieri e parole, e se con una rapida irruzione il narratore spezza il filo del racconto con una congettura (Ibid: 22), è al registro del "verosimile" che affida la preparazione dell'atmosfera emotiva carica di tensione che deve condurre alle pagine della retata: "verso le 4 del mattino, la sparatoria si placò. Faceva freddo, l'umidità della notte piovosa attraversava i muri. Nella levataccia, tutti erano rimasti in camicia e ciabatte, con appena qualche scialletto o pastrano sulle spalle. I letti abbandonati avevano forse custodito un po' di tepore. Stanchi, con quel senso di cavo e di disseccato che lascia dentro le orbite una grossa emozione, con le ossa peste, battendo i denti, ciascuno tornò alla sua casa, nel proprio letto" (Ibid). Poi la retata, alle cinque di mattina, che il narratore chiama "ora psicologica" su cui i testimoni discordano e concordano al contempo, quando i testimoni odono una ragazza, Letizia detta "l'Occhialona", gridare "Oh Dio, i mamonni!" (Ibid: 23). La cronaca rientra nel vivo, e il narratore diventa il regista di una sequenza che somiglia a un filmato in presa diretta: "noi seguiteremo a parlare del Ghetto, perché fu l'epicentro della razzia", e poco oltre dice "entriamo ora in una casa di via S. Ambrogio, nel Ghetto. Potremo seguire la razzia in tutte le sue fasi" (Ibid: 24-25). Il racconto stringe sulla prospettiva di un'unica testimone, la signora S., che racconta come "il dramma entrava nella vita, vi si mescolava con una spaventosa naturalezza, che lì per lì non lasciava campo nemmeno allo stupore" (Ibid: 26): il romanzesco, autorizzato come strumento per penetrare negli interstizi fra $i$ fatti e connetterli in un quadro 
che assomigli alla vita, finisce con il distruggere il romanzo, con il negarlo alla base dicendo che il dramma diventa la vita stessa, che quello che sembra un intreccio romanzesco fu vita reale, al punto che decadde fra gli ebrei perfino lo stupore. Per vie diverse, con intenti teorici e partendo da premesse estetiche del tutto diverse, Manzoni e Debenedetti davanti alla realtà decretano la medesima sentenza di morte per il romanzo, la medesima impossibilità per la sua compiuta riuscita davanti a una realtà che si sottrae alla volontà dell'autore di ridurla entro uno schema, un ordine, una giustificazione. I miti, se mai, subentrano dopo, a cose fatte, quando si può senza affanno imbastire un racconto, farsi una ragione di cose che, nel loro accadere, non ne hanno.

Come se di fronte all'insensatezza della realtà la fiducia nella parola poetica si fosse incrinata, in queste due opere di assiste all'avvenuta lacerazione fra l'assentimento logico e razionale al vero e il desiderio di apprendere il dolore e il male della storia in modo obliquo attraverso la finzione e il suo potere consolatorio. Tuttavia, il fatto che Manzoni e Debenedetti, per motivi differenti, perseguano un'idea di letteratura che scuote alle fondamenta la pratica narrativa dell'invenzione e che fa del verosimile un mezzo retorico per intaccare la superficie compatta della realtà, non significa che essi cessino di credere nel potere della letteratura e della lingua letteraria. Le loro opere esprimono un ideale di crudeltà di cui non si compiacciono perché, parafrasando Adorno, il crudele è il momento della "autoriflessione critica" dell'arte letteraria che "davanti allo strapotere della realtà non può più presumere a priori di saper trasformare il terribile in forma" (Adorno 2009: 68). La crudeltà non si esibisce nelle sue forme esteriori ma rimane implicita nelle parole, poche e disperse, delle vittime, alle quali i due autori rimettono il vero potere di innescare la riflessione critica:

L'uno e l'altro [Piazza e Mora] sopportarono quel lungo supplizio, quella serie e varietà di supplizi, con una forza che, in uomini vinti tante volte dal timor della morte e dal dolore; in uomini i quali morivan vittime, non di qualche gran causa, ma d'un miserabile accidente, d'un errore sciocco, di facili e basse frodi; in uomini che, diventando infami, rimanevano oscuri, e all'esecrazion pubblica non avevano altro da opporre che il sentimento di un'innocenza volgare, non creduta, rinnegata tante volte da loro medesimi; in uomini [...] che avevano una famiglia, moglie, figliuoli, non si saprebbe intendere, se non si sapesse che fu rassegnazione: quel dono che, nell'ingiustizia degli uomini, fa vedere la giustizia di Dio, e nelle pene, qualunque siano, la caparra, non solo del perdono, ma del premio. L'uno e l'altro non cessaron di dire, fino all'ultimo, fin sulla rota, che accettavan la morte in pena de' peccati che avevan commessi davvero (Manzoni 2004: 126).

La reazione critica all'ingiustizia degli uomini si rovescia, nel credente Manzoni, nella consolante fede in una giustizia che, benché non in questo 
mondo, esiste ed è stabile, eterna, certa. La parola, come sua figura, riceve una parte del suo vigore e della sua verità, ma non pretende più di esserne rivelazione riportando il brutto a una qualche forma di armonia o di ordine. Che gli uomini oscuri del processo, morti nell'infamia, conosceranno una gloria maggiore lo dice la fede, ma la letteratura non può testimoniare tale redenzione bensì solo rinfocolarne la speranza. In Debenedetti nemmeno più questo margine persiste: “"che faranno di noi?' Queste povere parole sono tra le poche lasciateci da coloro nell'andarsene. Ci fanno sentire la voce di un essere tornato per un momento nella nostra vita, tra noi, quando a lui la nostra vita qui non apparteneva più, e già era entrato in quella nuova esistenza oscura e terribile" (Debenedetti 2001: 30). Le vittime sono, se possibile, ancora più oscure di quelle del processo, il loro viaggio, iniziato in modo arbitrario e inspiegabile, e condotto nell'indifferenza di giovani soldati tedeschi che, con l'occasione, fanno i "turisti" davanti al Vaticano, finisce letteralmente nel nulla con "un invito al silenzio, a non tentare più di rimetterli nel consorzio umano [...] l'ultima parola, l'ultimo segno di vita che ci sia giunto da loro" (Ibid: 48).

Consapevole del proprio ruolo di intellettuale, Debenedetti lascia un'opera di spirito illuministico che rifugge ogni compiacimento letterario, pur non disdegnando l'eleganza della prosa, e soprattutto non inserisce nel dramma collettivo l'individualità tragica del personaggio: non "bisogna credere che la tragedia si sia svolta in un'atmosfera di muta e trasecolata solennità" (Ibid: 26), e nella descrizione di un drammatico incontro fra $\mathrm{i}$ tedeschi e una famiglia ebrea su un pianerottolo, scrive il narratore che "il tragico, l'intensità, la complicazione dei movimenti che stanno per avvenire su questo pianerottolo, potrebbero far pensare a uno spazio adeguato, si starebbe per dire eschileo: il che non risponderebbe al vero" (Ibid: 33). Il tragico sta nel rifiuto di credere all'avvertimento di Celeste, nel non voler vedere e nel percorrere la via verso un destino di annientamento che secondo Debenedetti è inscritto nella storia d'Israele da sempre. Mancano invece il pathos della tragedia, il palco allestito, le pose, la gravezza solenne. Ciò che profondamente accomuna 16 ottobre 1943 a Storia della colonna infame è quindi l'intuizione che la rappresentazione del male e dell'ingiustizia non preveda la scena della tragedia coi suoi eroi nobili e grandi, ma lo spazio dimesso e comune della realtà quotidiana di ogni tempo e di ogni giorno. La sua banalità consiste, come aveva intuito Manzoni, in un errore logico in cui la ragione tende in seguito a riposare, accecata dall'amor proprio o da altre passioni (la paura, la pigrizia, l'egoismo ecc.), anziché contrastarlo ed emendarlo (Manzoni 1963: 354-355). Non è lecito quindi muovere a Manzoni e Debenedetti accuse di "fariseismo": essi non biasimano le vittime dell'ingiustizia per non aver fatto fronte alla minaccia con il martirio o con la fuga, bensì, al contrario, mostrano senza alcuna ambiguità coloro che di 
quel male portano la colpa: i giudici che per trovare colpevoli gli imputati, "per respingere il vero che ricompariva ogni momento, in mille forme, e da mille parti, con caratteri chiari allora com'ora, come sempre, dovettero fare continui sforzi d'ingegno, e ricorrere a espedienti, de' quali non potevano ignorar l'ingiustizia" (Manzoni 2004: 5); ai tedeschi e ai loro tirapiedi fascisti che nelle poche ore della razzia e della degradante detenzione nel Collegio Militare in attesa della deportazione espressero con chiarezza "il proposito di umiliare, di deprimere, di ridurre quella gente a stracci umani, senza più una volontà, quasi senza più rispetto di se stessi [...]" (Debenedetti 2001: 46-46). In entrambi i casi i due autori denunciano attraverso la letteratura la vera abiezione dell'ingiustizia, il vero scandalo del male: non il fatto che $\mathrm{i}$ giudici e i nazisti fecero morire degli innocenti, ma che "per quanto dipendeva da loro" li fecero "morir colpevoli" (Manzoni 2004: 125).

\section{BIBLIOGRAFIA}

Adorno, T. (2009). Teoria estetica. Torino: Einaudi.

Benjamin, W. (2004a). Destino e carattere. In Angelus novus. 8a ed. (pp. 31-38). Torino: Einaudi.

Benjamin, W. (2004b). Le affinità elettive. In Angelus novus. 8a ed. (pp. 163-234). Torino: Einaudi.

Berardinelli, A. (1996). La forma del saggio. In F. Brioschi e C. Di Girolamo (a cura di). Manuale di letteratura italiana. 4 voll. (vol. 4, pp. 809-885). Torino: Bollati Boringhieri.

Borghesi, A. (1989). La lotta con l'angelo. Giacomo Debenedetti critico letterario. Venezia: Marsilio.

Ciocchetti, M. (2006). Prima di piantare datteri. Giacomo Debenedetti a Roma (1944-1945). Pesaro: Metauro.

Debenedetti, G. (1994a). “Campo d'ebrei [1945]”. In Saggi critici. Terza serie (pp. 78-83). Venezia: Marsilio.

Debenedetti, G. (1994b). Personaggi e destino [1947]. In Saggi critici. Terza serie. Venezia: Marsilio (pp. 111-127).

Debenedetti, G. (2001). 16 ottobre 1943. Torino: Einaudi.

Manzoni, A. (1963). Osservazioni sulla morale cattolica. Parte prima [1819]. In Tutte le opere. 7 voll. (vol. 3, pp. 263-480). Milano: Mondadori.

Manzoni, A. (1981a). Lettera a Monsieur Chauvet sull'unità di tempo e di luogo nella tragedia [1820]. In Scritti di teoria letteraria (pp. 59-153). Milano: Rizzoli.

Manzoni, A. (1981b). Del romanzo storico e, in genere, de' componimenti misti di storia e d'invenzione. In Scritti di teoria letteraria (pp. 193-282). Milano: Rizzoli. 
Manzoni, A. (1986). Tutte le lettere. 3 voll. Milano: Adelphi.

Manzoni, A. (2004). Storia della colonna infame. In Storia della colonna infame. Vol. 12 dell'Edizione nazionale ed europea delle opere di Alessandro Manzoni (pp. 1-160). Milano: Centro Studi Manzoniani.

Negri, R. (1972). Il romanzo-inchiesta del Manzoni. Italianistica, 1, 14-42. Raimondi, R. (2004). La ferita del passato. Alessandro Manzoni, Storia della colonna infame. In Letteratura e identità nazionale (pp. 67-123). Milano: Bruno Mondadori.

Riccardi, C. (1990). Il "reale" e il "possibile". Dal "Carmagnola" alla "Colonna infame”. Firenze: Le Monnier.

Ricoeur, P. (1986). Tempo e racconto. 3 voll. Milano: Jaca Books.

Wittgenstein, L. (2011). Della certezza. L'analisi filosofica del senso comune. Torino: Einaudi.

\author{
CHRONICLE, HISTORY AND LIKELYHOOD. \\ 16 OTTOBRE 1943 AND STORIA DELLA COLONNA INFAME
}

\title{
Summary
}

This article proposes a comparative analysis of 16 ottobre 1943 (1944) by Giacomo Debenedetti and Storia della colonna infame (1840) by Alessandro Manzoni. The premise consists in considering Manzoni's text as a model used by Debenedetti for his brief chronicle of the razzia in the ghetto of Rome. The comparison is first of all carried out as an analysis of the narrative rhetoric. The connection between Debenedetti's and Manzoni's works is found in the theoretical speculation of the latter about the relationship between truth and likelyhood in history and literature. The second point of comparison lies in the issue of destiny, where the two authors diverge: while Manzoni conceives destiny as an aspect of Providence and finds in faith a consolation to suffering and injustice, Debenedetti acknowledges evil only as absurd and blind chance. The theoretical and aesthetic consequence is that both the authors reject novel as a literary form no longer fit to organize the destiny of a character into the weft controlled by the narrator. Therefore, they combine history, chronicle and likelyhood into a hybrid narrative that does not justify evil through the closed narrative order but rather investigates its origin and moral meaning.

Keywords: Manzoni, Debenedetti, Non-fiction, history and literature, ethics and literature, theory of likelyhood. 